\title{
Technique for the development of technological assembly tooling at the stage of product design
}

\author{
Mikhail Kovalevich, Elena Shemonaeva*, and Alexey Goncharov \\ Moscow Aviation Institute (National Research University), 4, Volokolamskoe highway, Moscow, \\ 125993, Russia
}

\begin{abstract}
The paper considers the solution of the problem of parallel design on the example of products of the aviation industry. A method for designing assembly technological equipment at the stage of conceptual design of a product is proposed. The scope of possible work, the relationship between the initial data and the scope of elaboration of the design of the assembly device are determined.
\end{abstract}

\section{Introduction}

Product lifecycle management is an effective way to reduce the time spent on product release. Technological preparation of production is an important stage of the life cycle, on the quality of the passage of which the success of the production of high-quality products largely depends. One of the most laborious and critical stages of technological preparation of production is the design of special technological equipment.

Traditionally, tooling design begins after the completion of the design stage and the receipt of approved design documentation, which significantly increases the time to start production.

One of the ways to reduce the terms of technological preparation of production is parallel design of the product and assembly technological equipment. At the stage of the draft design, as a rule, there is already data that will allow you to perform a significant amount of work on the design of assembly fixtures. A prerequisite for the implementation of parallel design is the effective exchange of up-to-date product data, which is possible only in digital production and with the support of product lifecycle management [1-4].

The paper considers the solution of the problem of parallel design by the example of products of the aviation industry.

\section{Design analysis}

Aircraft product designs are generally not self-sufficient in terms of assembly. Due to their low rigidity, basing on structural elements, which is typical for general mechanical engineering, does not allow obtaining the required high assembly accuracy.

*Corresponding author: shemonaevaes@mati.ru 
In this regard, assembly devices are widely used, which, first of all, perform the function of additional assembly bases [5-6].

The assembly fixture consists of the following main elements:

- contour basic elements designed to ensure the accuracy of conformity of the product contour to the contour of the outer contour;

- point base elements for basing on holes;

- the frame of the assembly device, which links all the elements into a single whole;

- various fixing, including clamping elements, which are necessary to prevent accidental movement of elements of the assembled structure;

- assembly elements of assembly devices for installation of basic elements on the frame.

In turn, high requirements are imposed on the accuracy of manufacturing the assembly devices themselves, which requires the use of special installation methods.

\section{Determination of initial data for design}

When the development of a product, for example, an aircraft, is at the stage of a draft design, the following initial data can already be obtained for the design of an assembly fixture:

- theoretical contour of the product (outer surface);

- scheme of dividing the aircraft into separate units with their overall dimensions;

- preliminary structural and structural diagram showing the approximate location of the structural elements of the airframe;

- approximate mass of both units and aircraft structure as a whole;

- additional data on the conditions of production and the possibilities of manufacturing technological equipment (for example, the availability of large-sized equipment);

Thus, when designing technological equipment in parallel with the stage of preliminary design of a product, the following necessary initial data remain undefined:

- the exact location of the frame elements;

- dimensions of individual structural elements.

In the end, only some of the elements of the assembly fixture can be designed finally, and some of the elements will have a preliminary view. The composition and relationship of the initial data and the designed elements of assembly devices are shown in Figure 1.

\section{Development of a design methodology}

The elements of the assembly device, which have a preliminary view, can be finalized only after the transfer of the approved design documentation for the product to production. Let's define a methodology for preliminary design of such elements.

\subsection{Designing the frame of the assembly fixture}

The frame design sequence includes the following steps:

- layout definition;

- determination of the dimensions of columns, beams, frames and other elements;

- assessment of the load on the frame elements with the subsequent determination of the assortment of semi-finished products for its manufacture;

- choice of methods of connection of frame elements.

The design of both the frame as a whole and its elements depends on the mass and dimensions of the product being assembled, as well as on the method of mounting the device, which, in turn, depends on the production capabilities. 


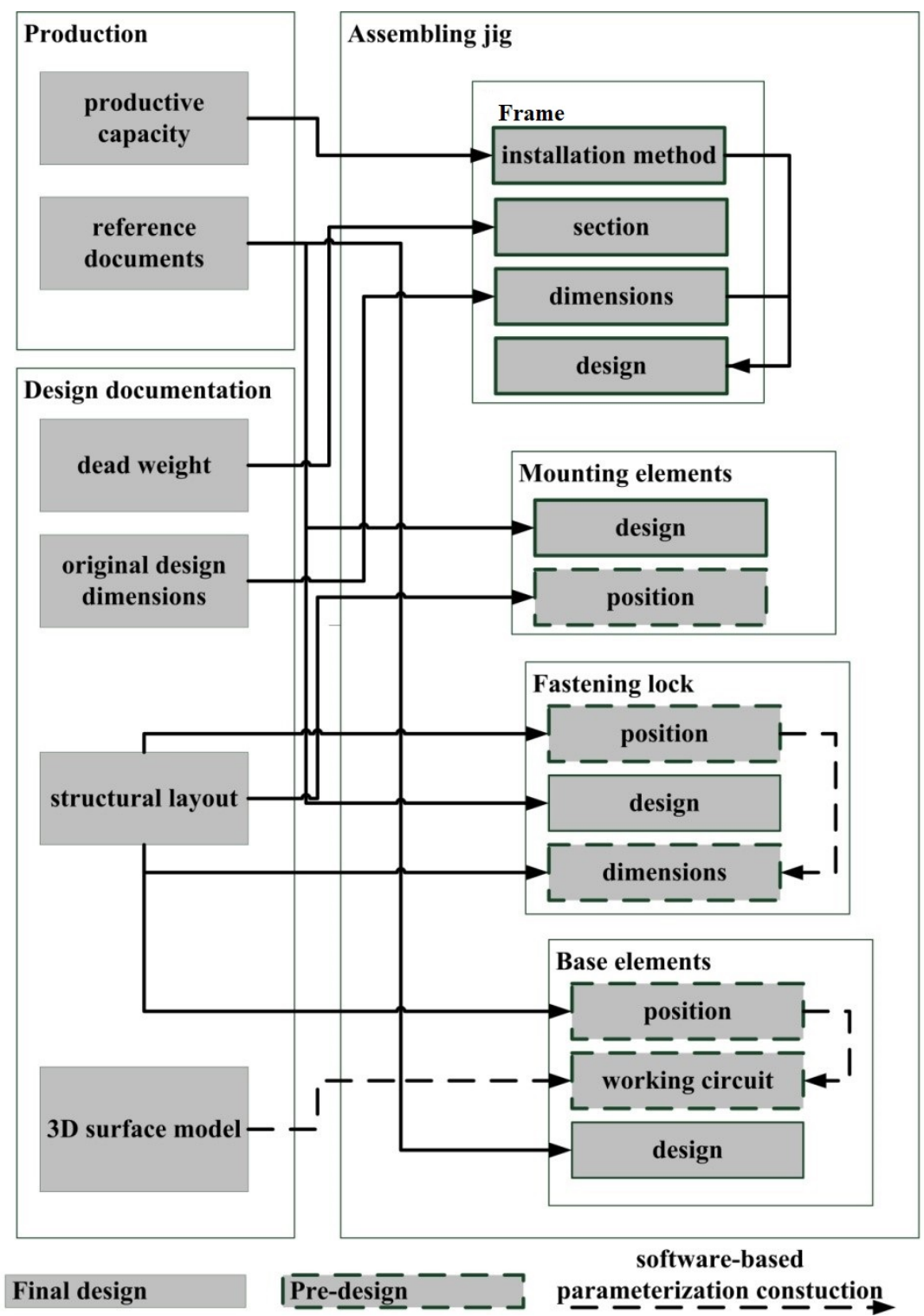

Fig. 1. The composition and relationship of the initial data and the designed elements of assembly devices

\subsection{Design of mounting elements}

It is necessary to develop the design of the mounting elements, as well as determine their location on the frame.

The design of the basic elements depends on the method of installation of the assembly device and within the framework of the chosen method, as a rule, their designs are unified. The location of the assembly elements is directly related to the location of the basic elements of the assembly fixture, and, therefore, affects the design of the frame elements of the assembly fixture. 


\subsection{Designing contour base elements}

The design sequence for basic elements includes:

- selection of a preliminary design solution;

- construction of the working contour and determination of the position of the mounting holes.

Contour basic elements are rigidly linked to the shape of the assembled product. Changing the position of the switch or cradle will generally lead to a change in the geometry of the working circuit. In this case, the design, if possible, should be such that when the position of the base element itself is changed, the position of the mounting holes on it does not change.

In addition, in a number of cases, the location and shape of the parts of the assembled product significantly affects the design of the basic elements due to the possibility of their intersection and the need to make various cuts in the basic element.

Thus, the contour basic elements have the greatest design uncertainty at the stage of the draft design. The parameterization of constructions can significantly reduce the complexity of making changes.

\subsection{Designing fastening lock}

The design sequence for point retainers includes:

- selection of a preliminary design solution;

- linking the location of the hole in the retainer with the expected location of the base hole in the part.

The design of point clamps should be largely standardized, and during the design process, their location and length change, depending on the location of the base hole in the part.

It is also advisable to compensate for the uncertainty of the retainer design by parametrizing the constructions.

\section{Development of a parameterization method}

The finalization of the pre-designed elements of the assembly fixture should be carried out as soon as possible. Based on this condition, parametrization of electronic geometric models of the designed elements of the assembly device can be used as a tool to reduce costs in the design. Modern 3D modeling systems such as Siemens NX have these features.

Within the framework of the problem being solved, the greatest effect is achieved with the parameterization of the following constructions:

- the working circuit of the switch, depending on its position relative to the based surface;

- the linear dimensions of the retainers, depending on the spatial location of the fixation points and the place of fastening of the retainer on the frame;

- the location of the holes on the frame of the assembly device, depending on the design and location of the mounting elements.

An example of the implementation of parameterization (2) when simulating the operating circuit of a knife switch (3), depending on its position relative to the grounded surface (1), is shown in Figure 2.

Parameterization is carried out as a result of linking the surface of the aircraft, mated with the switch, the switch circuit and its location. This binding is performed using the tools of the Wave module of the Siemens NX software package. The Wave Geometric Relationship Editor tool allows you to take geometry of solids, faces, curves, and datum 
planes (as well as variables) from a parent part and reference it in a child part, creating inter-model relationships. Thus, the top-down design method is implemented. This method allows you to rebuild the parts automatically, without the intervention of the designer - if the plane of the switch is moved, the body of the switch will be automatically changed in accordance with the skin surface and its boundaries and the position of the base plane. The Constraint Browser tool displays inter-model relationships (parameter mapping) in an assembly, allowing you to track related part features. An example of such a relationship is shown in Figure 3.

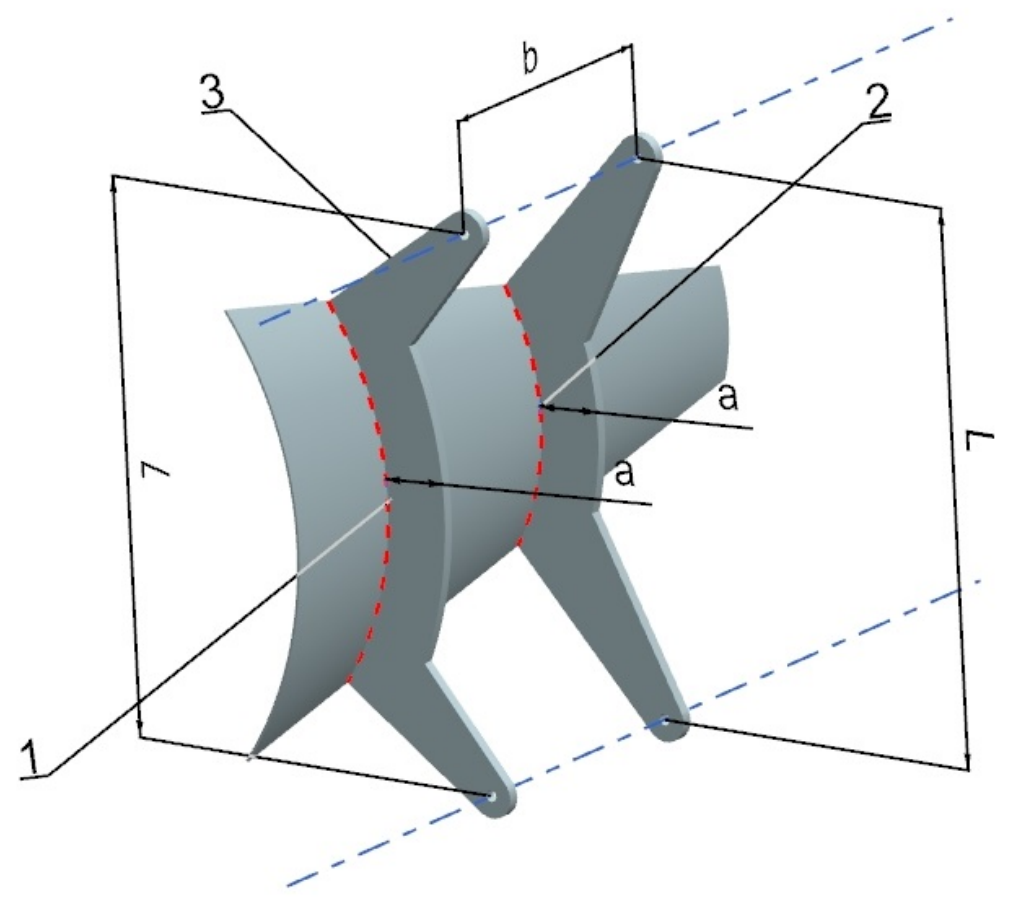

Fig. 2. Parametrization of the operating circuit of the knife switch depending on its position relative to the reference surface.

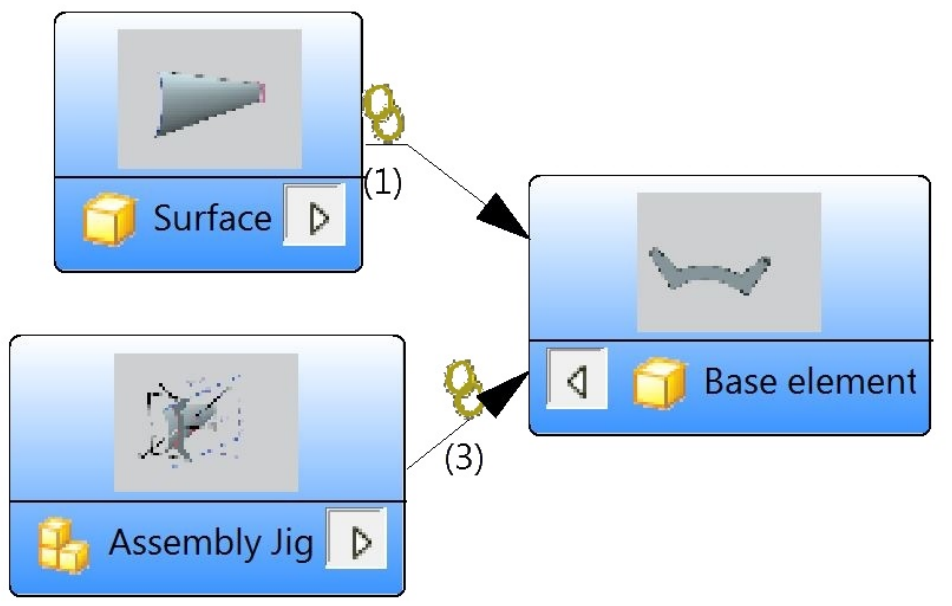

Fig. 3. Parameter binding scheme in an assembly. 


\section{Conclusion}

In a flexible digital manufacturing environment, with proper organization of product lifecycle management, a significant part of the technological preparation of production can be performed in parallel with the main design of the structure.

The use of parallel design will reduce the period of the stage of technological preparation of production several times.

The use of parametrization significantly reduces the complexity of designing technological equipment at the stage of finalizing the electronic model.

Further research in this direction can be aimed at creating fully parameterized universal electronic models of technological equipment and assessing the effectiveness of their application.

\section{References}

1. F. Mas, J.L.Menendez, M.Oliva, A. Gomez, J.Rios Collaborative Engineering Paradigm Applied to the Aerospace Industry. In: Bernard A., Rivest L., Dutta D. (eds) Product Lifecycle Management for Society. PLM 2013. IFIP Advances in Information and Communication Technology, v. 409, pp.675-684 (2013)

2. F. Mas, J.L.Menendez, M.Oliva, J.Rios, Collaborative Engineering: an Airbus case study, Procedia Engineering, v. 63, pp. 336-345 (2013)

3. Z. Chen, J. Tang, Aircraft digital assembly process design technology based on $3 D$ Model MATEC Web Conf. 20202004 (2018)

4. H. Zhang, L. Zheng, X. Chen, H. Huang, A novel reconfigurable assembly jig based on stable agile joints and adaptive positioning-clamping bolts, Procedia Cirp, v.44, pp. 316-321 (2016)

5. A.I. Pekarsh, U.M. Tarasov, G.A. Krivov, Modern technologies for aircraft assembly (Moscow, Agraf-press, 2006)

6. R.I. Guseva Features of the airframe assembly technology (Komsomol'sk-na-Amure, FGBOU VPO «KnAGTU», 2013) 